\title{
Prevalence of angioid streaks and other ocular complications of Paget's disease of bone
}

\author{
T R Dabbs, K Skjodt
}

\begin{abstract}
Seventy randomly selected patients with Paget's disease of bone were examined for ocular complications. The prevalence of macular degeneration and cataract was $24.3 \%$. Only one patient was found to have angioid streaks. Eight patients had peripapillary chorioretinal atrophy. These findings suggest that the prevalence of serious ocular complications of Paget's disease is not as high as previously thought. The significance of peripapillary chorioretinal atrophy requires further evaluation.
\end{abstract}

Paget's disease has been recognised as having ocular sequelae since James Paget's original description of the disorder in 1877. 'The complications may be divided into two main groups; those intrinsic to the eye, and those resulting from compression of the orbital contents or sensory and motor nerves supplying the eye and adnexae. The intrinsic disorders reported are corneal opacities, cataract, angioid streaks, and disciform macular degeneration. Those conditions due to compression include papilloedema, optic atrophy, extraocular muscle palsies, exophthalmos, and nasolacrimal duct obstruction. ${ }^{2}$ The mechanisms by which compressive lesions exert their effects are easily understood but not so the intrinsic lesions. From the ophthalmological standpoint angioid streaks are the lesions which attract most attention because of their potential for producing sight-threatening subretinal neovascular membranes, which are treatable in appropriate circumstances. ${ }^{3}$ Accurate data on the prevalence of these ocular complications and in particular angioid streaks are obviously of importance in the management of Paget's disease.

Angioid streaks were originally described by Doyne in $1889 .{ }^{+}$Paget's disease was first observed as an association by Terry in $1934 .^{5}$ After studying 50 cases he identified four with 'definite angioid streaks'. From this finding he concluded the 'The coexistence of angioid streaks and osteitis deformans is of too frequent occurrence to be the result of chance alone'. Several reports have since confirmed the presence of angioid streaks in cases of proved Paget's disease. The prevalence has been placed at somewhere between $8 \%$ and $15 \% .{ }^{67}$ The only recent survey undertaken which had the advantage of employing fluorescein angiography to detect angioid streaks was carried out by Clarkson and
Altman in 1982. ${ }^{*}$ They examined 50 selected sufferers from Paget's disease and performed fluorescein angiography on every patient, discovering five cases of angioid streaks, placing the prevalence at $10 \%$.

Necropsy evidence of Paget's disease suggests an overall prevalence of $3 \%$ in the population aged over 40 , rising to $9 \%$ in those aged over $80 . .^{410}$ From these figures it has been estimated that there are approximately 750000 people in the UK with Paget's disease. This would suggest that the number with angioid streaks is between 60000 (8\%) and 112500 (15\%).

In view of these findings Paget's disease might be expected to account for a considerable number of patients with ocular disease, particularly macular degeneration. But on clinical grounds Paget's appears to feature very infrequently as a cause of ocular problems in everyday practice. In order to evaluate the prevalence and nature of retinal and other ocular disorders in Paget's disease a study of randomly selected patients was undertaken.

\section{Patients and methods}

The patients were recruited from inpatients and outpatients attending the Regional Metabolic Unit at the Royal Hallamshire Hospital, Sheffield. The group of patients approached were attending for the purposes of a separate investigation into their past exposure to canine distemper. All patients were offered an ophthalmic examination and were advised about the aims of the project. Seventy patients were included in the study. All had radiologically proved Paget's disease, the diagnosis being confirmed by iliac crest bone biopsy. Every patient had pain, with or without deformity, and was receiving treatment accordingly.

Each patient was interviewed and a full history obtained. An ocular examination was undertaken, including refraction slit-lamp biomicroscopy, tonometry, confrontation fields, and funduscopy after pupillary dilatation. When a significant retinal abnormality was discovered, fluorescein angiography was performed. The risks involved in performing angiography in asymptomatic subjects without identifiable retinal lesions was considered to be unjustifiable. Sixteen patients underwent fluorescein angiography, and all were reviewed separately by two ophthalmologists. The diagnosis of angioid streaks was made according to the criteria 
Table 1 Comparison of patients with and without peripapillary atrophy

\begin{tabular}{llll}
\hline & With PPA $(n=8)$ & Without PPA $(n=61)$ & Significance \\
\hline Age mean (SD) & $71 \cdot 98(8 \cdot 98)$ & $71 \cdot 88(9 \cdot 55)$ & NS $^{\star}$ \\
Duration & $16(1-46)$ & $6(0 \cdot 16-30)$ & NS $\dagger$ \\
Median (range) & $2 \cdot 75(1 \cdot 5-5 \cdot 5)$ & $2(1-8)$ & NS $\dagger$ \\
Average VA & &
\end{tabular}

^Two-sample $t$ test. + Wilcoxon rank sum test.

$\begin{array}{llllllllllll}\text { †VA } & 6 / 6 & 6 / 9 & 6 / 12 & 6 / 18 & 6 / 24 & 6 / 36 & 6 / 60 & \text { CF } & \text { HM } & \text { PL } & \text { NPL } \\ \text { Code } & 1 & 2 & 3 & 4 & 5 & 6 & 7 & 8 & 9 & 10 & 11\end{array}$

Table 2 Prevalence of macular degeneration and cataract

\begin{tabular}{llll}
\hline & $\begin{array}{l}\text { Total } \text { no. of } \\
\text { eyes affected }\end{array}$ & $\begin{array}{l}\text { Disease in one } \\
\text { or both eyes }\end{array}$ & $\begin{array}{l}\text { Prevalence } \\
\%\end{array}$ \\
\hline Cataract & 31 & 17 & $24 \cdot 28$ \\
Macular degeneration & 25 & 17 & $24 \cdot 28$ \\
\hline
\end{tabular}

described by Shields et al. "Accordingly, linear, variably coloured lesions present at the level of Bruch's membrane were being sought during fundus examination. The fluorescein angiograms were scrutinised for the presence of directly fluorescing streaks or those showing hyperfluorescent borders during the later phases of angiography.

Statistical analysis was performed with the MINITAB software package and comprised the $\chi^{2}$ test for categorical variables and the twosample $t$ test for continuous variables. When continuous variables were skewed, the nonparametric Wilcoxon rank sum test was employed.

\section{Results}

Seventy patients were entered into the study. There were equal numbers of males and females. The age range was 49 to 88 years, mean 71.6 years. The duration of disease ranged from two months to 46 years, mean 8.2 years.

The sites affected were divided into four categories: pelvis, head, axial skeleton, and limbs. The number of patients with one affected region was 33 , two sites 20 , and three sites, 17. No patient had disease in all four sites.

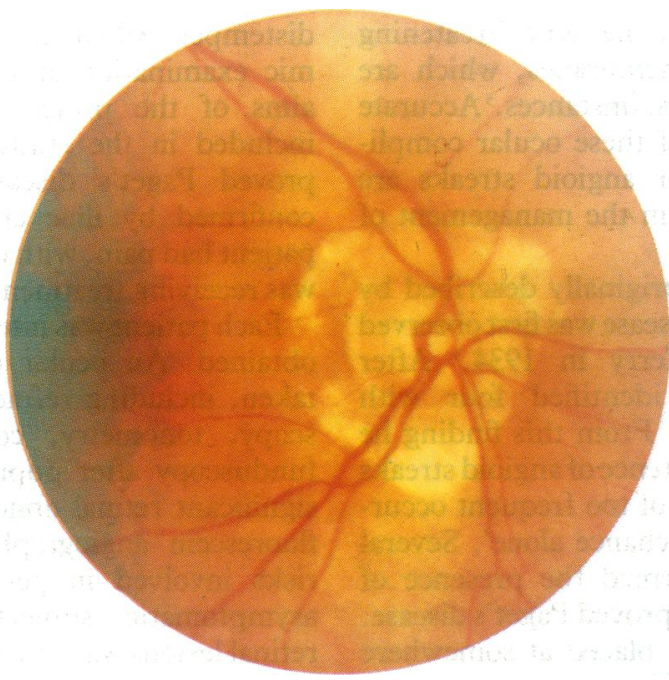

Figure 1A

Figure 1 Colour photograph $(A)$ and a fluorescein angiogram $(B)$ of the right eye of a patient with peripapillary atrophy. The hyperfluorescent area visible inferior to the disc was not regarded as sufficiently well defined to qualify as an angioid streak. This subject is an 82-year-old woman with Paget's disease of 46 year' duration affecting three principal sites.
Visual acuity was noted and divided into 11 groups as shown in Table 1. In order to compare the visual performance of our group with those of other epidemiological studies the prevalence of macular degeneration and cataract was evaluated in accordance with the criteria adopted by the Framingham Eye Study in the following ways. ${ }^{12}$ The presence of cataract judged to account for a visual acuity of $6 / 9$ or less was recorded. Macular degeneration was identified as the 'dry' type, such as pigment disturbance or drusen formation, and of the exudative type with elevation of the retinal pigment epithelium or neurosensory retina. If these were detected, senile macular degeneration was diagnosed provided the visual acuity was $6 / 9$ or worse.

Macular degeneration and cataract were found to be equally prevalent, as shown in Table 2 . In only one case was the visual acuity reduced sufficiently to warrant blind registration; the cause being bilateral cataract.

The retinal findings were as follows:

Peripapillary chorioretinal atrophy. This was seen in 16 eyes of eight patients. Six of these patients underwent fluorescein angiography; two refused this test. The choriocapillaris and the retinal pigment epithelium appeared to be deficient in the immediate peripapillary zone, leading to hyperfluorescence on fluorescein angiography. The width of this hyperfluorescent ring was judged to be abnormally large. A typical example of peripapillary atrophy is shown in Figs 1 A, B.

This group of patients with peripapillary atrophy was compared with the rest of the study population with regard to age, visual acuity, and duration of Paget's disease. No statistically significant differences could be detected. An attempt was made at correlating the number and type of sites affected with the presence of peripapillary atrophy, but the number of cases was too small to show any statistically viable associations. It is, however, notable that of patients having the disease for more than 15 years (15 of 70) 1/3 were accounted for by patients with peripapillary atrophy (Table I).

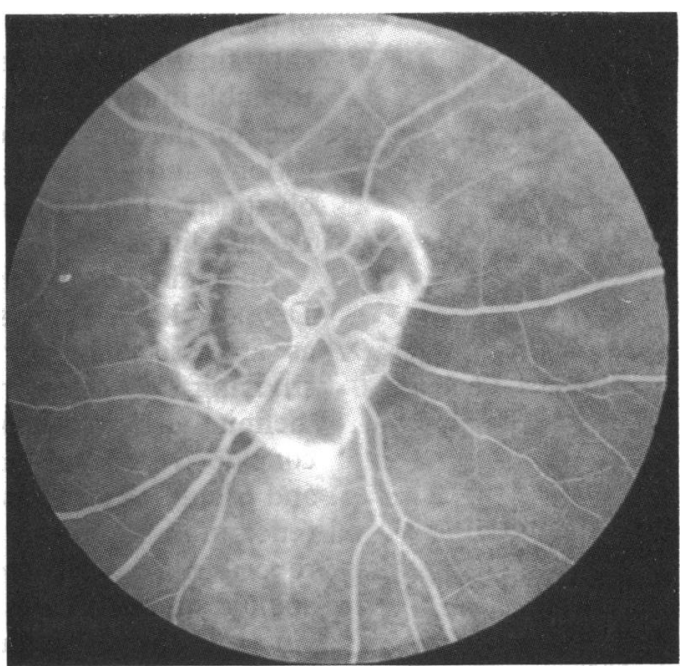

Figure 1B 


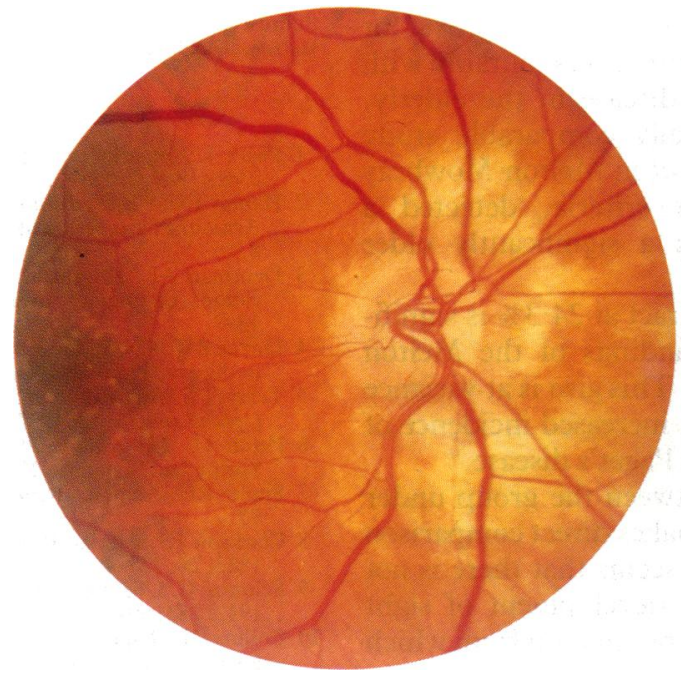

Figure 2A

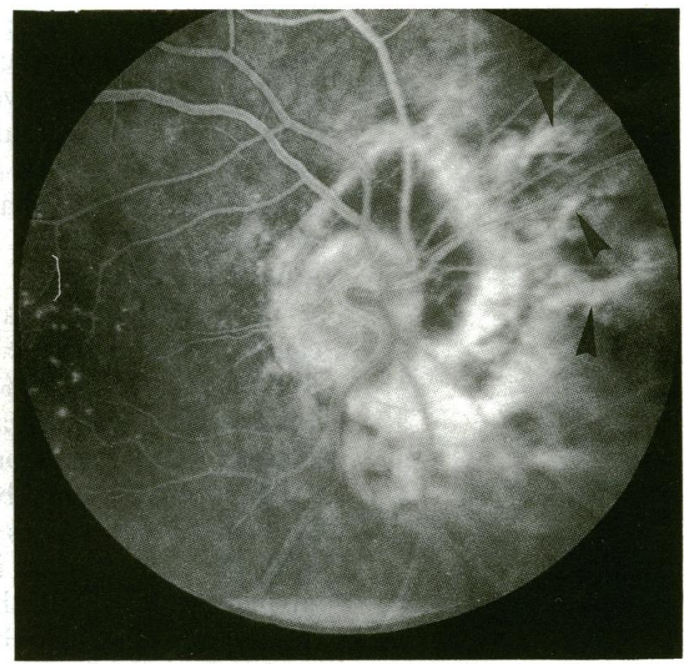

Figure $2 B$

Figure 2 Colour photograph $(A)$ and a fluorescein angiogram $(B)$ of the right eye of the single patient judged to have angioid streaks. The hyperfluorescent linear lesions radiating from the ring of peripapillary atrophy, though atypical, were thought to represent individual streaks (arrowed).

Angioid streaks. Both eyes of one patient showed marked peripapillary chorioretinal atrophy with linear extentions radiating a short distance peripherally (Figs $2 \mathrm{~A}, \mathrm{~B}$ ). Although not typical, the angiographic appearance was thought to be consistent with angioid streaks. This patient also had macular drusen and atrophic macular degeneration affecting both eyes, the left being more severely affected. The patient, a man, was 69 and had had Paget's disease for eight years; it affected his pelvis, spine, and both legs. His visual acuities were 6/9 in the right eye and $6 / 60$ in the left. He had no signs of pseudoxanthoma elasticum; a skin biopsy was not performed. This one patient represents an overall prevalence of $1.4 \%$ for angioid streaks in the series under study.

Peau d'orange was not identified in any case studied.

Compression of ocular cranial nerves was not identified, but one patient had considerable distortion of one orbit leading to downward displacement of the left globe. Fortunately there was no associated diplopia or optic nerve compression. Other conditions causing visual problems were chronic open-angle glaucoma in one patient, amblyopia in one eye, persistent untreatable retinal detachment in another, and bilateral cystoid macular oedema following intracapsular cataract surgery in a further patient. No cases of corneal dystrophy were discovered.

\section{Discussion}

The principal finding of this study was the low prevalence of angioid streaks $(1 \cdot 4 \%)$. The explanations probably lies in the method of patient selection and examination. Our group was randomly selected, and the severity of their disease was variable. In their survey Clarkson and Altman ${ }^{8}$ selected patients with severe disease. Earlier studies may have tended to include patients with more advanced disease, as the increased availability of radiography and biochemical analysis now allow for earlier detection of Paget's disease.
With the exception of the studies by Terry ${ }^{5}$ and Clarkson and Altman ${ }^{8}$ none have been carried out by one investigating team on a group of Paget's sufferers; the reported prevalences were arrived at by collecting cases from several sources, thus potentially leading to inaccurate estimates. $^{67}$

Peripapillary chorioretinal atrophy has been described in other conditions associated with angioid streaks and is regarded as a precursor of angioid streaks. ${ }^{71}$ The only previous estimate of the frequency of peripapillary atrophy was that of Terry at $18 \% .^{5}$ We detected it in eight out of 70 $(11 \cdot 5 \%)$.

The definition of peripapillary atrophy is somewhat inexact. Fluorescein angiography is of little help, as there is no information as to the extent of normal peripapillary hyperfluorescence. Peripapillary changes occur in myopia, presumed ocular histoplasmosis, and peripapillary choroidal sclerosis, and are said to occur as a consequence of aging. "113

None of our patients were myopic, nor were there any signs of presumed ocular histoplasmosis.

Choroidal sclerosis is regarded as being difficult to separate from the peripapillary changes in angioid streaks, and according to Krill and Archer peripapillary choroidal sclerosis may progress to angioid streaks, further indicating a considerable degree of overlap between the two conditions. ${ }^{11+}$ Some of our patients may therefore have been cases of peripapillary choroidal sclerosis, or in some cases their condition may have been due to age alone.

We attempted to identify any factors separating patients with peripapillary atrophy from the rest of the subjects, but no statistically significant result was forthcoming.

One-third of the patients with a disease duration of 15 years or more were accounted for by this group. Peripapillary atrophy might be related to the duration of Paget' disease, but a larger study is needed to prove this statistically.

Our subjects did not have an excess of serious 
ocular disease. The prevalence of $24 \cdot 3 \%$ for macular degeneration compares favourably with previous studies of eye disease in the elderly. The most demographically comparable study carried out by Gibson et al in Melton Mowbray using the Framingham criteria, detected a prevalence of $38.8 \%$ in a significantly older group of patients. ${ }^{15}$

The prevalence of cataract at $24.3 \%$ is significantly lower than the findings of the Melton Mowbray study (41.6\%). This also is at variance with earlier reports of an increased incidence of cataract in sufferers from Paget's disease. ${ }^{16}$

The age difference between the group under study and other studies makes direct comparison difficult. Nevertheless it seems that there is not an increased risk of the development of sight threatening disease in the population from which our study group was drawn.

We conclude that Paget's disease is only infrequently associated with angioid streaks, and confers no excess risk of serious eye disease. The existence of peripapillary atrophy was noted in eight patients and is probably related to the duration of the disease. This relationship requires further study for clarification.

We thank Dr J A Kanis for allowing us to study his patients, Mr J Gibson and Mr J Talbot for their help with interpretation of the fluorescein angiograms and $\mathrm{Mr} \mathrm{D}$ Shaw for undertaking the statistical analyses.

1 Paget J. Remarks on osteitis deformans. Illustrated Medical News 1989; 2: 181-2.

2 Hamdy RC. Paget's disease of bone: assessment and management. Armour Pharmaceutical Company (Praeger, UK), 1981: 57 -

3 Deutman AF, Kovaks B. Argon laser treatment in complications of angioid streaks. Am $\mathcal{F}$ Ophthalmol 1979; 88: 13-7.

4 Doyne RW. Choroidal and retinal changes - the result of blows to the eye. Trans Ophthalmol Soc UK 1889; 9: 128-40.

5 Terry TT. Angioid streaks and osteitis deformans Trans Am Ophthalmol Soc 1934; 32: 555-73.

6 Paton D. The relation of angioid streaks to systemic disease. Springfield: Thomas, 1972;20-41.

7 Sholtz RO. Angioid streaks. Arch Ophthalmol 1941; 26: 677

8 Clarkson JG, Altman RD. Angioid streaks. Surv Ophthalmol $1882 ; 26: 235-46$

9 Schmorl G. Über Osteitus deformans Paget. Virchows Arch $(A)$ 1932; 283: 694-751.

10 Collins DH Paget's disease of bone - incidence and subclinical forms. Lancet 1956; ii: 51-7.

11 Shields JA, Federman JL, Tomer TL, Annesley WH. Angioid streaks: 1. Ophthalmoscopic variations and diagnostic problems. Br f Ophthalmol 1975; 59: 257-64.

12 Khan HA, Liebowiz HM, Ganley JP, et al. The Framingham Eye Study. 1. Outline and major prevalence findings. Am $\mathcal{f}$ Eye Study. 1. Outline and major prevalence findings. Am Epidemion 1977 ; $106: 17-32$.

. Hagerstown: Harper and Row, 1988; 4: chapter 66: 1-2.

14 Krill AE, Archer D Classification of the choroidal atrophies. Am F Ophthalmol 1971; 72: 562-85.

15 Gibson JM, Rosenthal AR, Lavery J. A study of the prevalence of eye disease in the elderly in an English community. Trans Ophthalmol Soc UK 1985; 104: 196-203.

16 Zion VM, Billet E. Clinical ophthalmology. Hagerstown: Harper and Row, 1988; 5: chapter 29: 13-5. 